\title{
Blue, green and red emissive silver nanoclusters formed in organic solvents
}

\author{
Isabel Díez ${ }^{* a c}$, Mykola I. Kanyuk ${ }^{b}$, Alexander P. Demchenko ${ }^{b}$, Andreas Walther $^{a d}$, Hua Jiang $^{a}$, Olli \\ Ikkala $^{a}$ and Robin H. A. Ras* ${ }^{* a}$
}

Received (in $X X X, X X X)$ Xth $X X X X X X X X X 20 X X$, Accepted $X$ th $X X X X X X X X X 20 X X$

DOI: $10.1039 / b 000000 x$

Strongly luminescent silver nanoclusters with tunable emission are directly synthesized in organic polar and apolar 10 solvents. We show that an amphiphilic polystyrene-blockpoly(methacrylic acid) block copolymer can be universally used as their support medium. A remarkable similarity in spectroscopic properties is observed between these clusters and charge-transfer organic dyes.

${ }_{15}$ Few-atom silver nanoclusters are fluorophores with an attractive set of properties including sub-nanometer size, high quantum yield and photostability. ${ }^{1-6}$ Being of much smaller size than semiconductor quantum dots and exhibiting nontoxicity and lack of blinking ${ }^{1}$ they are attractive for biosensor applications, 20 biological imaging, ${ }^{7,} 8$ optoelectronic devices, ${ }^{9}, 10$ chemical sensing ${ }^{11-13}$ and optical recording media. ${ }^{14,15}$ Since the first report on stable fluorescent silver clusters in solution ${ }^{16}$ much attention has been paid to optimize and tune their emission properties and stability by varying the type of scaffold and synthesis procedure.

${ }_{25}$ Silver nanoclusters are usually encapsulated in water-soluble, polar, organic scaffolds that provide multiple binding sites for silver including polymers, ${ }^{17-21}$ dendrimers, ${ }^{16,22}$ peptides $^{23,}{ }^{24}$ and DNA oligonucleotides. ${ }^{1,} 3,25-28$ A common feature of silver nanoclusters in organic scaffolds is that their synthesis is carried 30 out in water as solvent. A few reports describe silver nanoclusters dispersed in polar organic solvents such as alcohols, however, these clusters were synthesized first in water, and subsequently transferred to the other solvent, either by mixing the aqueous solution with the organic solvent, ${ }^{29}$ by precipitation from water 35 followed by redissolution ${ }^{19}$ or by phase transfer. ${ }^{30,31}$ On the other hand, inorganic glasses ${ }^{15,32}$ and silver nanoparticles ${ }^{33}$ have been

${ }^{a}$ Department of Applied Physics, Aalto University School of Science (previously Helsinki University of Technology), P.O.Box 15100, FIN40 00076, Espoo, Finland. E-mail: robin.ras@aalto.fi

${ }^{b}$ A. V. Palladin Institute of Biochemistry, 9 Leontovicha st., 01030 Kiev, Ukraine.

${ }^{c}$ Present Address: Departamento de Física de la Materia Condensada, ICMA-CSIC, Facultad de Ciencias, Universidad de Zaragoza, 50009 45 Zaragoza, Spain. E-mail: isabeldiez@unizar.es

${ }^{d}$ Present Address: DWI at the RWTH Aachen, 52056 Aachen, Germany

$\dagger$ Electronic Supplementary Information (ESI) available: materials, synthesis and characterization. See DOI: 10.1039/b000000x/

$\ddagger$ We thank Markus Linder, Myhailo Losytskyy and Valentyna Tokar for 50 providing access to the fluorescence spectroscopic instrumentation.
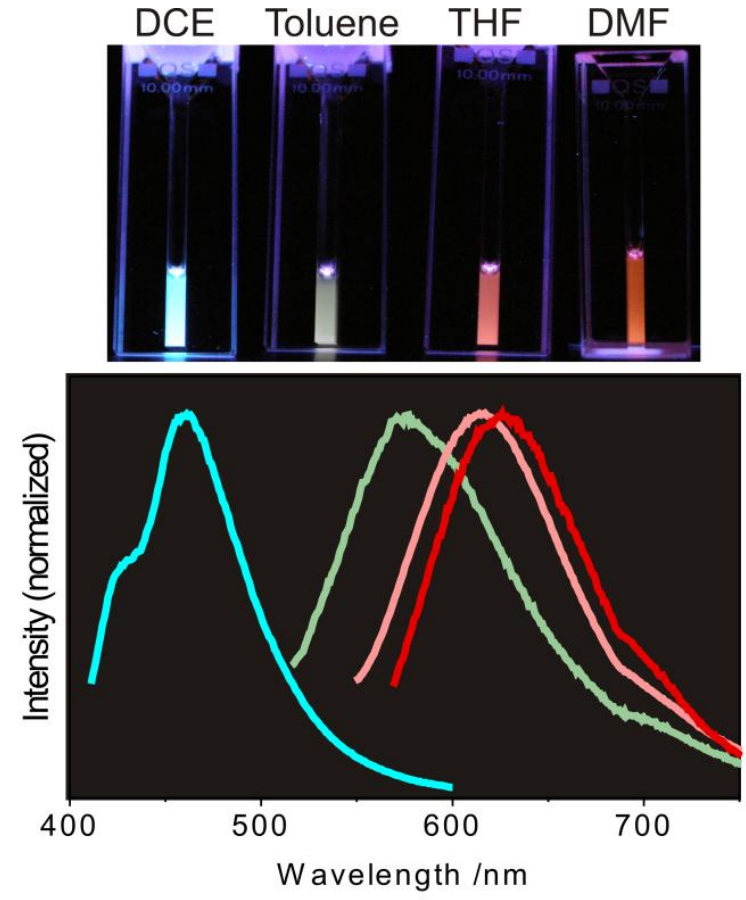

Fig. 1. Photographs and corresponding emission spectra of polymer-supported silver clusters in various solvents: 1,2dichloroethane (DCE), toluene, tetrahydrofuran (THF) and dimethylformamide (DMF).

55 shown as suitable support for silver nanoclusters in absence of water. This raises the question whether organic scaffolds would allow formation of silver clusters also in non-aqueous solvents.

In this study we present the first recipe to synthesize fluorescent silver nanoclusters directly in polar and apolar 60 organic solvents without the use of water. We show that local environments formed by the polymer in these solvents allow formation of brightly emissive silver clusters of which the fluorescence output is strongly solvent-dependent. The scaffold for the formation of silver clusters is an amphiphilic polystyrene${ }_{65}$ block-poly(methacrylic acid) block copolymer (PS- $b$-PMAA) $\left(\mathrm{PS}_{52}-\mathrm{PMAA}_{48}, \mathrm{M}_{\mathrm{w}}=74 \mathrm{kDa}\right)$. The PMAA homopolymer was 

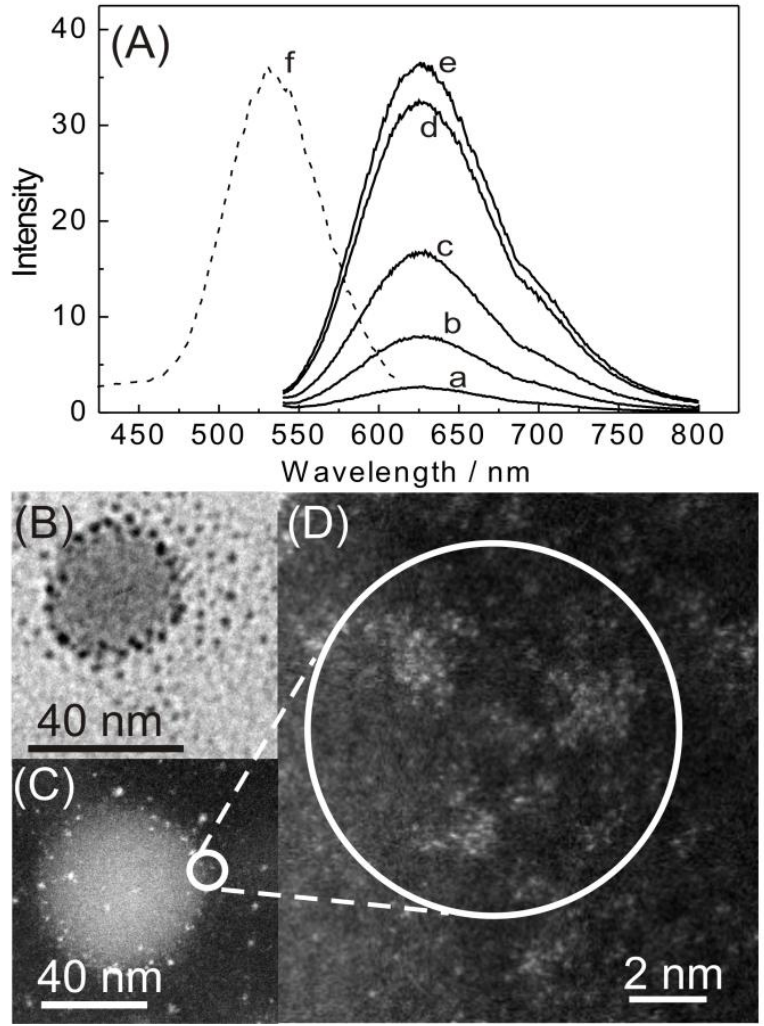

Fig. 2. (A) Emission (a-e) and excitation (f) spectra of silver clusters formed in DMF with increasing irradiation time: (a) 1, (b) 4, (c) 8, (d) 12 and (e) 16 hours. (B) TEM and (C) spherical aberration 5 corrected HAADF STEM images of the same sample after 8 hours of irradiation time. (D) Higher magnification of a region in image (C).

already reported to be an excellent template for silver clusters in aqueous medium. ${ }^{17,19,31,34}$ In the current work the PS block was 10 selected to extend the solubility to polar and apolar organic solvents. The silver precursor are organo-soluble silver salts such as $\mathrm{AgClO}_{4}$ or $\mathrm{AgPF}_{6}$. The clusters were synthesized under identical overall concentrations and molar ratios of silver to methacrylic acid segments. After mixing the block copolymer 15 with the silver salt in selected solvents, the mixtures were irradiated with visible light while controlling the appearance of fluorescence. The strong luminescence of the silver nanoclusters solutions is shown in Fig. 1, top. Different solvents allow tuning the fluorescence across the visible spectrum (Fig. 1, bottom).

20 During photoactivation, the emission intensity increases gradually with irradiation time. On initial steps this change is linear with subsequent observation of tendency to saturation. Essentially, the positions of the spectra remain unchanged with irradiation time, indicating that during the photoactivation the 25 number of silver clusters increases without affecting the type of major part of emitters. For instance, in Fig. 2A we show the results of this observation for a sample prepared in DMF.

In studied solvents the polymer molecules combined with silver form micellar structures (Figure S4). Figure 2B shows the 30 bright-field low-resolution TEM image of a single micelle having
Table 1. Photoluminescence data of silver clusters. Positions of excitation $\left(\lambda_{\max }^{\mathrm{ex}}\right)$ and emission $\left(\lambda^{\mathrm{em}}{ }_{\max }\right)$ maxima, Stokes shifts $(\Delta \mathrm{v})$, widths of emission bands $\left(\Delta_{1 / 2}\right)$ and quantum yields $(\Phi)$.

\begin{tabular}{cccccc}
\hline Solvent & $\lambda_{\text {max }}^{\mathrm{ex}}(\mathrm{nm})$ & $\lambda^{\mathrm{em}}{ }_{\max }(\mathrm{nm})$ & $\Delta v\left(\mathrm{~cm}^{-1}\right)$ & $\Delta_{1 / 2}\left(\mathrm{~cm}^{-1}\right)$ & $\Phi(\%)$ \\
\hline DCE & 365 & 420 & n.d. & n.d. & 33 \\
Toluene & 490 & 574 & 2990 & 3120 & - \\
THF & 515 & 599 & 2720 & 3300 & - \\
Water & 535 & 613 & 2380 & 2663 & - \\
DMF & 534 & 626 & 2750 & 2470 & 24 \\
\hline
\end{tabular}

35 a PS-core (about $40 \mathrm{~nm}$ in diameter), and a PMAA-shell containing clearly observable dots smaller than $2 \mathrm{~nm}$. Spherical aberration corrected high angle annular dark field scanning transmission electron microscopy (HAADF STEM) (Fig. 2C) shows comparable features at low magnification. On the other 40 hand, higher magnification allows imaging the arrangement of silver in the micelle shell with resolution approaching atomic scale (Fig. 2D). The dots of about $2 \mathrm{~nm}$ visible at low magnification (Fig. 2C, circle) are identifiable at higher magnification as non-crystalline assemblies of silver atoms (Fig. ${ }_{45} 2 \mathrm{D}$, circle). Although being very close to each other, the silver atoms do not form a crystalline nanoparticle, which demonstrates their effective stabilization by the PMAA chains. Comparable assemblies of silver atoms were as well reported for silver nanoclusters prepared in water with PMAA homopolymer. ${ }^{31}$ The

50 mass of the clusters could not be determined using mass spectrometry because the high molecular weight polymer, tightly bound to the clusters, cannot be detected.

Previously we had shown that silver clusters in PMAA homopolymer dispersed in polar solvents exhibit moderate 55 solvatochromism, i.e. solvent-dependent shifts of the spectrum. ${ }^{19}$ solvatochromism was also studied for silver clusters prepared in ssDNA. ${ }^{29}$ Here we demonstrate significant larger shifts when the nanoclusters are made in low-polarity solvents (Fig. 1). For instance, in polar solvents such as DMF and THF, red emissive 60 silver clusters are formed, of which the emission spectra are comparable to our previous work on nanoclusters in PMAA homopolymer in water solution. ${ }^{19}$ For an apolar solvent such as toluene, the clusters emit in the green region, and in DCE the emission is centred in the blue. The estimated quantum yields in ${ }_{65}$ DCE and DMF (Table 1) are remarkably larger, 6-fold, than reported for silver clusters formed in aqueous solutions of the homopolymer (PMAA). ${ }^{19}$ Samples in THF and toluene are also highly luminescent but turbidity does not allow determination of their quantum yield.

70 The use of different solvents leads to significant differences in fluorescence properties of the silver clusters. Short-wavelength excitation/emission wavelengths of $365 / 420 \mathrm{~nm}$ for DCE contrasts the shifted to longer wavelengths positions of these bands at 490/574 $\mathrm{nm}$ for toluene and further long-wavelength 75 shifted positions at $515 / 599 \mathrm{~nm}, 535 / 613 \mathrm{~nm}$ and $534 / 626 \mathrm{~nm}$ in THF, water and DMF correspondingly (Table 1).

We checked if fluorescence spectra can be excited in the UV region and discovered bands that closely resemble the characteristic high-energy absorption bands of organic dyes. The ${ }_{80} \mathrm{UV}$ excitation band for silver clusters in DMF is located at 320 

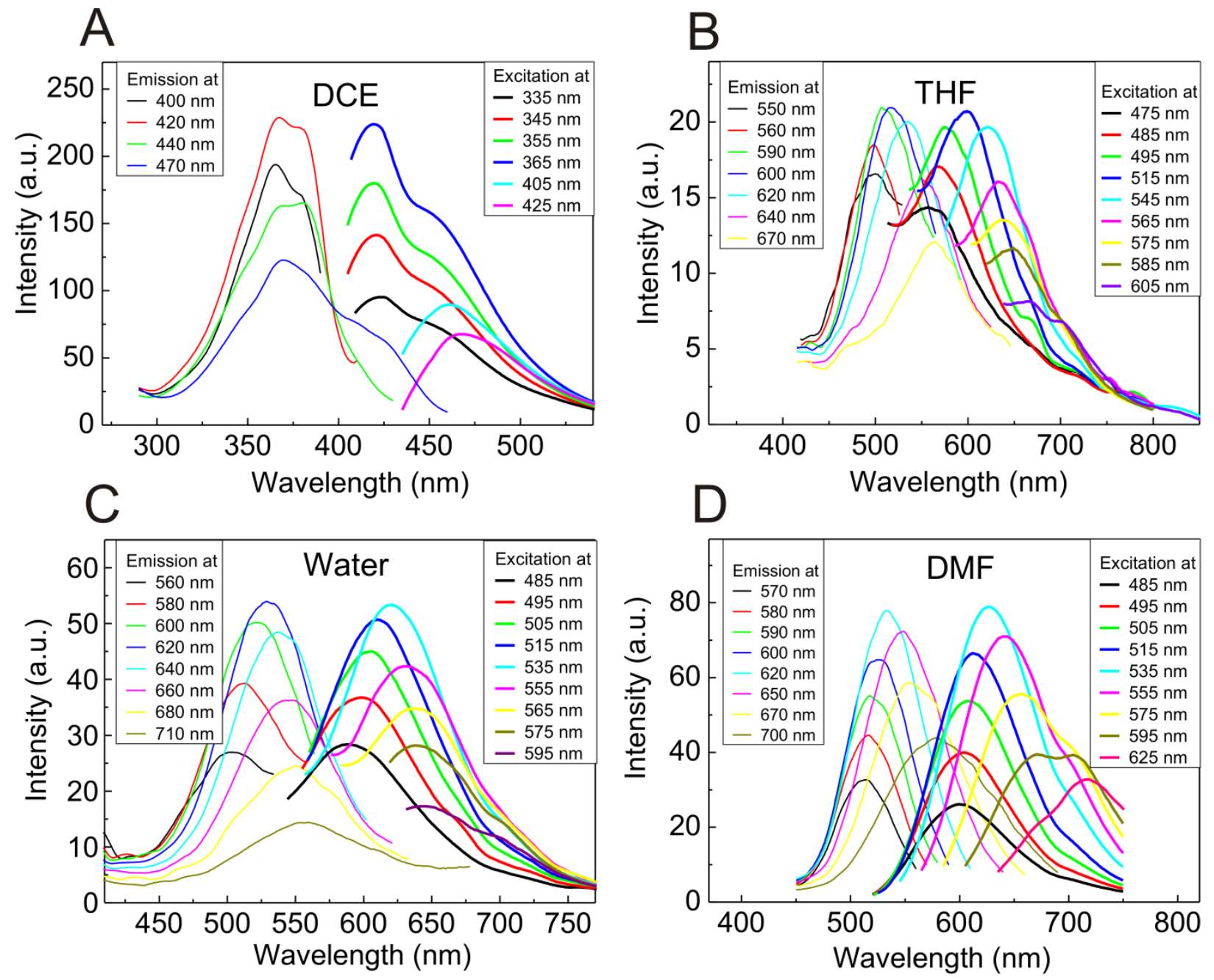

Fig. 3. Fluorescence excitation spectra at fixed emission wavelengths (left) and fluorescence emission spectra at fixed excitation wavelengths (right) for silver clusters formed on block copolymer in (A) DCE, (B) THF, (C) water and (D) DMF.

$5 \mathrm{~nm}$. (see Supporting Info). When excited by UV light the fluorescence bands are located at exactly the same positions as obtained by common excitation in the visible. To the best of our knowledge, this is the first report showing that the Kasha rule (i.e. ultra-rapid relaxation to the lowest excited-state level and the 10 emission only from this level), which is common for organic dyes, is also valid for silver nanoclusters. Several publications on fluorescent silver clusters in water reported on the large shift of emission band position as a function of excitation wavelength and it was explained by structural heterogeneity of formed clusters. ${ }^{17}$, ${ }_{15}{ }^{20,22,27}$ Although the cluster size remains unknown we show here that silver clusters with different optical properties can be synthesized without changing the scaffold, just by varying the nature of the solvent. And since we are able to record undistorted excitation spectra for clusters formed in different solvents, this 20 important issue was studied in detail. For the case of DCE we observe the presence of discrete bands both in excitation and emission with clear possibility of their photoselection by variation of excitation wavelength or emission wavelength, indicating the presence of such heterogeneity (Fig. 3A). In all 25 other cases smooth and symmetrical dominant components are observed that are mirror-imaged between excitation and emission bands (Fig. 3, B-D). By gradual variation of emission wavelength there occurs the gradual shift of excitation bands, and vice versa by the gradual variation of excitation wavelength a gradual shift 30 of emission bands is seen. Previously such effects were observed in fluorescence of organic dyes under very specific conditions: the presence of equally strong transition dipole moments in the ground and excited states causing distribution of their interactions with dielectric environment and slow mobility of this 35 environment making this distribution persistent on a time scale of emission. $^{35}$ Typical for such heterogeneity causing "inhomogeneous broadening" of spectra are the strong Stokes shifts and solvatochromism that we also observed here. Therefore 
the analogy in photophysical behaviour of presently studied clusters and organic dyes can be much stronger than it was previously thought. In view of the presence of electron-rich oxygen atoms in the stabilizing carboxylic acid group, we may 5 suggest the formation of ligand-to-metal charge transfer (LMCT) complexes. Similar idea was already suggested to explain the behaviour of $\mathrm{Au}$ nanoclusters. ${ }^{36} \mathrm{We}$ believe that the polymer conformation depends on the solvent providing different environments for the cluster formation. Formed in different 10 environments, the clusters might have different structure and exhibit different interaction with the environment. Several discrete maxima in spectra (as in DCE) might be due to variations in composition, whereas in spectra of the same symmetrical shape (as in DMF and water) the molecular environment may influence 15 the energies of electronic transitions causing the spectral shifts or solvatochromism. Meantime we believe that mechanistically the excited states are the same, showing LMCT. This state is strongly dipolar and interacts with surrounding dipoles producing a distribution in energies due to variability of dipole-dipole 20 interactions. This makes the excitation and emission contours symmetric and the excitation-dependent changes of emission spectra as well as the emission-dependent changes of excitation spectra gradual.

In conclusion, we present the first and universal recipe to 25 synthesize highly fluorescent silver clusters directly not only in water, but also in polar and apolar organic solvents, in a very simple way, using photoactivation of a solution of block copolymer and silver salt. The amphiphilic block copolymer plays a very important role being the template for preparing the 30 silver clusters and enabling their stabilization in various organic solvents. The choice of the organic solvent determines the emission wavelength of the clusters, covering a wide region of the spectrum from red via green to blue. The fluorescence is characterized by large, almost solvent-independent Stokes shifts, 35 by spectral inhomogeneous broadening observed in excitation and emission and by a second excitation band in the UV region. This report on the water-free synthesis of strongly fluorescent silver clusters in even apolar solvents is anticipated to open pathways for a variety of novel applications of these attractive 40 fluorophores.

\section{References}

1. T. Vosch, Y. Antoku, J. C. Hsiang, C. I. Richards, J. I. Gonzalez and $45 \quad$ R. M. Dickson, Proc. Natl. Acad. Sci. USA, 2007, 104, 12616-12621.

2. N. de Souza, Nature Methods, 2007, 4, 540-540.

3. C. I. Richards, S. Choi, J. C. Hsiang, Y. Antoku, T. Vosch, A. Bongiorno, Y. L. Tzeng and R. M. Dickson, J. Am. Chem. Soc., 2008, 130, 5038-5039.

50 4. H. Xu and K. S. Suslick, Adv. Mater., 2010, 22, 1078-1082.

5. I. Díez and R. H. A. Ras, in Advanced fluorescence reporters in chemistry and biology II; Chapter: Few-Atom Silver Clusters as Fluorescent Reporters, ed. A. P. Demchenko, 2010, vol. 9, pp. 307332.

55 6. I. Díez and R. H. A. Ras, Nanoscale, 2011, 3, 1963-1970.

7. Y. Antoku, J. Hotta, H. Mizuno, R. M. Dickson, J. Hofkens and T. Vosch, Photochem. Photobiol. Sci., 2010, 9, 716-721.

8. C.-A. J. Lin, C.-H. Lee, J.-T. Hsieh, H.-H. Wang, J. K. Li, J.-L. Shen, W.-H. Chan, H.-I. Yeh and W. H. Chang, J. Med. Biol. Eng., 2009,

9. T. H. Lee, J. I. Gonzalez, J. Zheng and R. M. Dickson, Acc. Chem. Res., 2005, 38, 534-541.
10. L. A. Peyser, A. E. Vinson, A. P. Bartko and R. M. Dickson, Science, 2001, 291, 103-106.

65 11. L. Shang and S. Dong, Biosens. Bioelectron., 2009, 24, 1569-1573.

12. W. Guo, J. Yuan and E. Wang, Chem. Commun., 2009, 3395-3397.

13. G. Y. Lan, C. C. Huang and H. T. Chang, Chem. Commun., 2010, 1257-1259.

14. G. De Cremer, B. F. Sels, J.-i. Hotta, M. B. J. Roeffaers, E.

70 Bartholomeeusen, E. Coutiño-Gonzalez, V. Valtchev, D. E. De Vos, T. Vosch and J. Hofkens, Adv. Mater., 2010, 22, 957-960.

15. A. Royon, K. Bourhis, M. Bellec, G. Papon, B. Bousquet, Y. Deshayes, T. Cardinal and L. Canioni, Adv. Mater., 2010, 22, 52825286.

75 16. J. Zheng and R. M. Dickson, J. Am. Chem. Soc., 2002, 124, 1398213983.

17. L. Shang and S. Dong, Chem. Commun., 2008, 1088-1090.

18. J. Zhang, S. Xu and E. Kumacheva, Adv. Mater., 2005, 17, $2336-$ 2340.

80 19. I. Díez, M. Pusa, S. Kulmala, H. Jiang, A. Walther, A. S. Goldmann, A. H. E. Müller, O. Ikkala and R. H. A. Ras, Angew. Chem. Int. Ed., 2009, 48, 2122-2125.

20. K. S. Suslick and H. X. Xu, ACS Nano, 2010, 4, 3209-3214.

21. X. Wang, S. Xu and W. Xu, Nanoscale, 2011, 3, 4670-4675.

85 22. Z. Shen, H. Duan and H. Frey, Adv. Mater., 2007, 19, 349-352.

23. J. Yu, S. A. Patel and R. M. Dickson, Angew. Chem. Int. Ed., 2007, 46, 2028-2030.

24. I. Díez, H. Hahn, O. Ikkala, H. G. Börner and R. H. A. Ras, Soft Matter, 2010, 6, 3160-3162.

90 25. B. Sengupta, C. M. Ritchie, J. G. Buckman, K. R. Johnsen, P. M. Goodwin and J. T. Petty, J. Phys. Chem. C, 2008, 112, 18776-18782.

26. E. G. Gwinn, P. O'Neill, A. J. Guerrero, D. Bouwmeester and D. K. Fygenson, Adv. Mater., 2008, 20, 279-283.

27. J. T. Petty, J. Zheng, N. V. Hud and R. M. Dickson, J. Am. Chem. Soc., 2004, 126, 5207-5212.

28. B. Han and E. Wang, Anal. Bioanal. Chem., 2012, 402, 129-138.

29. S. A. Patel, M. Cozzuol, J. M. Hales, C. I. Richards, M. Sartin, J. C. Hsiang, T. Vosch, J. W. Perry and R. M. Dickson, J. Phys. Chem. C, 2009, 113, 20264-20270

100 30. T. U. B. Rao and T. Pradeep, Angew. Chem. Int. Ed., 2010, 49, 39253929.

31. I. Díez, H. Jiang and R. H. A. Ras, ChemPhysChem, 2010, 11, $3100-$ 3104.

32. V. K. Tikhomirov, T. Vosch, E. Fron, V. D. Rodriguez, J. J. 105 Velazquez, D. Kirilenko, G. Van Tendeloo, J. Hofkens, M. Van der Auweraer and V. V. Moshchalkov, RSC Advances, 2012, 2, 14961501.

33. L. Maretti, P. S. Billone, Y. Liu and J. C. Scaiano, J. Am. Chem. Soc., 2009, 131, 13972-13980.

110 34. I. Díez, P. Eronen, M. Österberg, M. B. Linder, O. Ikkala and R. H. A. Ras, Macromol. Biosci., 2011, 11, 1185-1191.

35. A. P. Demchenko, in Methods in Enzymology, eds. L. Brand and M. L. Johnson, Academic Press, 2008, vol. 450, pp. 59-78.

36. Z. Wu and R. Jin, Nano Lett, 2010, 10, 2568-2573. 